\title{
Oddities of Active Systems
}

\section{Odd Diffusivity of Chiral Random Motion}

Authors: Cory Hargus , Jeffrey M. Epstein, and Kranthi K. Mandadapu

Phys. Rev. Letters 127, 178001 (2021)

\section{Recommended with a Commentary by Alexander Y. Grosberg, Department of Physics and Center for Soft Matter Research, New York University, 726 Broadway, New York, NY 10011}

Textbook (for instance, [1]) says that mobility of a particle in a fluid and diffusion coefficient are related to one another by Einstein relation $D_{i j}=\mu_{i j} k_{B} T$, and each is a symmetric tensor: $D_{i j}=D_{j i}$ and $\mu_{i j}=\mu_{j i}$. Following Onsager, textbooks explain that the latter symmetry, although can be derived from hydrodynamics, is more fundamental and is due to the time reversal symmetry of the underlying molecular mechanics. All of this is of course well familiar. Furthermore, symmetry of the diffusivity tensor seems to survive quite drastic generalizations. For instance, active Brownian particles, on the length and time scales above their persistence length/time, perform the usual random walks (which is why they are called Brownian), and, therefore, obey the usual Fick's law.

As a reminder, active Brownian particles (see, e.g., [2]) are the ones which are propelled forward by some internal source of energy with a fixed speed $v_{0}$, but in the direction that slowly reorients due to angular diffusion. To keep things simple, in 2D these particles obey overdamped Langevin dynamics of the form

$$
\dot{\mathbf{r}}=v_{0} \mathbf{e}(t) \text {, with unit vector } \mathrm{e}=(\cos \theta(t), \sin \theta(t)) \text {, and } \dot{\theta}=2 D_{r} \xi(t) \text {, }
$$

where $\xi(t)$ is, as usual, zero mean and unit variance white noise. If there is a concentration gradient of these particles, it results in the flux $\mathrm{J}=-D \nabla c$, where $D$ can be thought of as a scalar, because $D_{i j}=D \delta_{i j}$. And this is true despite the fact that active Brownian particles are propelled and so are certainly driven out of equilibrium. Accordingly, Einstein relation with mobility is violated - although many authors follow the temptation to introduce some "effective temperature" to save the Einstein's relation (see, e.g., [3] as an example) - which is tempting and possible because mobility $\mu_{i j}$ and diffusivity $D_{i j}$ are both symmetric tensors.

But...

Consider now slightly modified model of active Brownian particles in 2D:

$$
\dot{\mathbf{r}}=v_{0} \mathbf{e}(t) \text {, with unit vector } \mathrm{e}=(\cos \theta(t), \sin \theta(t)), \text { and } \dot{\theta}=\omega_{0}+2 D_{r} \xi(t) \text {. }
$$

The newly added term $\omega_{0}$ in the last equation indicates that driving particles steer systematically (maybe just a little bit, but systematically) to one side more than to the other, for 
instance, they may prefer to turn right rather than left. That, of course, means that they tend to make circles.

This immediately reminds us about our textbooks: good ones warn us that Onsager symmetry requires modification in the presence of a magnetic field (leading to $\mu_{i j}(H)=$ $\left.\mu_{j i}(-H)\right)$ or a Coriolis force.

If one starts thinking about it naively, one may be tempted to conclude that this bias in steering should not affect the fact that particles should be transported from the region of high to the region of low concentration, meaning that concentration should still satisfy the familiar diffusion equation

$$
\partial_{t} c=D \nabla^{2} c
$$

- which is true.

Cory Hargus, Jeffrey M. Epstein, and Kranthi K. Mandadapu in their recent PRL (which is also included here in the list of references [4]) take a fresh and deeper look at this situation in its entirety, and come to several interesting conclusions. First conclusion, which gives the title to their article, is that diffusion coefficient is now not a symmetric tensor - despite the fact that equation (3) holds, with scalar $D$ ! What happens is that

$$
D_{i j}=\left[\begin{array}{cc}
D & -D^{\perp} \\
D^{\perp} & D
\end{array}\right],
$$

where $D^{\perp}$ is the new parameter (proportional to $\omega_{0}$ in formula (2) for the example of active Brownian particles). The antisymmetric part of the diffusion tensor indicates that there exists some flux which is perpendicular to the concentration gradient, for instance, $J_{x}=-D \partial_{x} c+D^{\perp} \partial_{y} c$ and $J_{y}=-D \partial_{y} c-D^{\perp} \partial_{x} c$ - but this "sideways" flux is, of course, divergence-free (because it is based on violating the detailed balance circling components of the trajectories) and thus it does not contribute to the continuity equation, which is why the diffusion equation (3) survives unchanged.

Along with active Brownian particles, authors consider also a simple (and illuminating) lattice model, where particles can move only along four directions on the square lattice, and also turn right a bit more frequently than left.

Clearly, the situation arises because both time reversal symmetry is broken (due to the drive) and left-right symmetry is broken (due to steering imbalance), and for this situation the results appear to be quite general.

Authors analyze further other aspects of this situation. Quite surprisingly, they discover, in particular, that the Green-Kubo relation, which expresses diffusion coefficient in terms of velocity-velocity correlation function, remains valid, while the relation between mobility and diffusion is no longer valid at all.

This phenomenon of odd diffusion is the latest addition to the collection of other "oddities" recently discovered for active systems, such as odd viscosity [5] and odd elasticity [6] (in fairness, odd viscosity was discussed in the literature long before, e.g., [7], but, having at the time no interesting applications, these studies had less impact than deserved). From a more general perspective, these works give a good illustration how the studies of novel objects originally motivated by, and now abstracted from, some biological systems, give rise to fruitful reexamination of the most basic concepts in statistical physics. 


\section{References}

[1] Masao Doi. Soft Matter Physics. Oxford University Press, 2013.

[2] A. P. Solon, M. E. Cates, and J. Tailleur. Active brownian particles and run-and-tumble particles: A comparative study. The European Physical Journal Special Topics, 224:1231 $-1262,2015$.

[3] I. Eshghi, J. A. Eaton, and A. Zidovska. Interphase chromatin undergoes a local sol-gel transition upon cell differentiation. Phys. Rev. Lett., 126:228101, 2021.

[4] Cory Hargus, Jeffrey M. Epstein, and Kranthi K. Mandadapu. Odd diffusivity of chiral random motion. Phys. Rev. Lett., 127:178001, 2021.

[5] Debarghya Banerjee, Anton Souslov, Alexander G. Abanov, and Vincenzo Vitelli. Odd viscosity in chiral active fluids. Nature Communications, pages 1573 - 1578, 2017.

[6] Colin Scheibner, Anton Souslov, Debarghya Banerjee, Piotr Surówka, William T. M. Irvine, and Vincenzo Vitelli. Odd elasticity. Nature Physics, 16:475 - 480, 2020.

[7] J. E. Avron. Odd viscosity. Journal of Statistical Physics, 92:543 - 557, 1998. 